\title{
Hepatitis por salmonellas
}

URES.: JOSE ZACARIAS *, PATRICIO ERJNK *.

El virus I $\mathrm{H}$ es el agente más importante en la etiología do la hepatitis en la población infantil de nuestro país, pero existen además una serie de otras causns de daño hepático que pueden simular el cutadro clínico de hcpatitis infecciosa. Entre ellos debemos mencionar otros agentes virales ( $\mathrm{SH}$, mononucleosis, inclusión citomegálica, etc.), hepatotóxicos (anestésicos, isoniazidas, tetracloruro de carbono, etc.), parasitarios y bacterianos. De este último grupo se conocen como causantes de daño hepático las Salmonellas tpyhi y paratyphi A, B y C. Se sabe que estos bacilos se localizan en diversos parénquimas y entre ellos en el hígado. Producen daño hepático que generalmente no se traduce en manifestaciones clínicas a excepción de hepatomegalia; (1) pero en ocasiones este daño es de tal magnitud que puede llevar a una insuficiencia hepática importante, Jlegando a veces a la etapa de coma hepútico como sucedió en dos de los cásos aquí presentados.

En la literatura nacional encontramos la excelentc revisión rcalizada por el Dr. J. Bauzá y G. Frascoli (2) que describen una incidencia de un $4,1 \%$ de hepatitis túficas y $13,6 \%$ de hepatitis parutíficas. Porcentaje semejante al descrito por Sepúlveda y Fuentes en 1946 (3). Orozco y col. (4) en 1959 en una revisión de fiebre tifoidea en adultos, encontró que la hepatitis clínica se observaba el $0,4 \%$ de las fiebres tifoidea y $0,6 \%$ de las fiebres paratíficas. Recientemente Link (6) publica 4 casos de hepatitis por Salmonella typhi. En la literatura extranjera aparecen ya descritos en la primera guerra mundial, focos epidémicos de hepatitis por Salmonella paratyphi; (7) posteriormente aparecen publicaciones de casos aislados de hepatitis túfica y de coma hepático de la misma etiología. (8-9).

\footnotetext{
- Hospital Calvo Mackenna.
}

Además de los tipos de Salmonellas ya mencionadas existen otras que son propias de los animales y que en determinadas circunstancias son patógenas para el hombre como las Salnonellas typhimurium, bareilly, newington, etc. Estas pueden también producir un cuadro de hepatitis semejante al de la hepatitis viral.

Hemos creído de interés presentar en esta ocasión la investigación realizada en 14 pacicntes con hepatitis por Salmonellas.

Materiai clínico. Se estudiaron 14 niños en un período comprendido entre Enero 1967 y Julio 1972. Siete de estos fueron diagnosticados en el año 1972, cuatro en 1971, 2 en 1967 y 1 en 1968.

Consideramos como hepatitis a todos aquellos pacientes con hepatomegalia, ictericia y/o transaminasas elevadas. El diagnóstico de Salmonellosis se hizo en base al cuadro clínico, alteraciones hematológicas, reacciones de aglutinación con los antígenos respectivos o por aislamiento del bacilo en los hernocultivos.

Resultado y comentakio. En la tabla No 1 se pueden observar las características clínicas de los pacientes estudiados.

Llama la atención el predominio de preescolares especialmente menores de 4 años. Todos presentaron fiebre y hepatomegalia y sólo en dos no se comprobó ictericia.

La espJenomegalia no fue un hallazgo frecuente. Dos pacientes presentaron hemorragia digestiva importante y sólo en dos casos se observó compromiso de conciencia asociado a signos de insuficiencia hepática.

En el estudio hematológico (tabla $N^{\circ} 2$ ) existió tanto leucopenia como leucocitosis predominando aquellos con número de leucocitos nor- 
T A B L A No 1

HEPATITIS POR SALMONELLA CARACTERISTICAS CLINICAS

\begin{tabular}{|c|c|c|c|c|c|c|c|c|c|c|c|c|c|c|}
\hline & 1 & 2 & 3 & 4 & 5 & 6 & 7 & 8 & 9 & 10 & 11 & 12 & 13 & 14 \\
\hline EDAD & $3 \% / 12$ & 29 & $2^{2 / 12}$ & $59 / 12$ & $5^{2} / 1$ & $3^{2} / 1$ & 13 & $16 / 1$ & $1 / 2$ & $06 / 2$ & $95 / 22$ & $43 / 12$ & $34 / 12$ & $32 / 2$ \\
\hline FIE BRE & + & + & + & + & + & + & + & + & + & + & $t$ & + & + & + \\
\hline ICTERICIA & + & $t+$ & $+t$ & ++ & $t+$ & ++ & + & + & + & $+t+$ & - & $++t$ & + & - \\
\hline $\begin{array}{l}\text { HEPATO- } \\
\text { MEGALIA }\end{array}$ & + & + & $+t$ & $+t$ & $+t$ & ++ & + & $t+$ & + & + & ++ & $+t$ & $t+$ & $+t$ \\
\hline $\begin{array}{l}\text { ESPLENO- } \\
\text { MEGALIA }\end{array}$ & - & - & - & - & $\rightarrow$ & + & + & $+t$ & - & $t$ & - & - & - & - \\
\hline $\begin{array}{l}\text { HEMO- } \\
\text { RRAGIA }\end{array}$ & - & - & + & + & - & - & - & - & - & - & + & + & - & - \\
\hline $\begin{array}{l}\text { COMPROM. } \\
\text { CONCIENCIA }\end{array}$ & - & & - & + & - & - & - & - & - & - & - & $++t$ & - & - \\
\hline
\end{tabular}

TA B L A NO 2

HEPATITIS PUR SALMONELLA ALTERACIONES HEMATOLOGICAS

\begin{tabular}{|c|c|c|c|c|}
\hline & $\begin{array}{c}\text { Gles. } \\
\text { Blancoss }\end{array}$ & $\begin{array}{l}\text { Eosing } \\
\text { filos \% }\end{array}$ & $\begin{array}{c}\text { Bacili- } \\
\text { formes } \%\end{array}$ & $\begin{array}{l}\text { Hemoglo } \\
\text { bina } \\
\text { g/ } 100 \mathrm{ml} .\end{array}$ \\
\hline 1 & 9.800 & 0 & 1 & 9.0 \\
\hline 2 & 7.400 & 2 & 8 & 7,8 \\
\hline 3 & 11.200 & 0 & 5 & 6.6 \\
\hline 4 & 6.700 & 2 & 40 & 12,3 \\
\hline 5 & 7.500 & 0 & 2 & 10,9 \\
\hline 6 & 9.300 & 0 & 28 & 11,4 \\
\hline 7 & 3.400 & 0 & 36 & 9.5 \\
\hline 8 & 8.300 & 0 & 20 & 6,2 \\
\hline 9 & 14.400 & 0 & 23 & 11,0 \\
\hline 10 & 5.700 & 0 & 38 & 11,0 \\
\hline 11 & 7.000 & 1 & 22 & 14.1 \\
\hline 12 & 19.200 & 1 & 10 & 10,9 \\
\hline 13 & 8.200 & 0 & 13 & 11,1 \\
\hline 14 & 8.700 & 0 & 59 & 10.9 \\
\hline
\end{tabular}

ma'es. La aneosinofilia y el aumento de baciliformes fue un hallazgo frecuente. Los casos que presentaron anemia intensa correspondieron a aquellos pacientes con hemorragias.
Las reacciones de aglutinación (tabla No 3 ) fueron negativas sólo en tres casos, dos de los cuales corresponden a una infección por Salmonella bareilly (cuyo anticuerpo no se pesquisa en estas reacciones de Widal clásico) y el otro a Salmonella paratyphi B. De los 11 casos en que se practicó hemocultivo, 6 de ellos fueron positivos.

Al estudiar la bilirrubinemia y transaminasa glutámica pirúvica (TGP) (tabla No 4) se encontró que dos tenían bilirrubinemia normal pero con TGP. elevada. Sólo 1 presentó TGP. normal con hiperbilirrubinemia que corresponde al caso No 1 que consultó después de 3 semanas con fiebre e ictericia. Llama la atención las cifras extremadamente altas tanto de TGP. como de bilirrubinemia que alcanzaron algunos pacientes.

Dos pacientes evolucionaron en forma tormentosa Hlegando a presentar signos de insuficiencia hepática grave y encefalopatía (coma hepático); las características clínicas y de laboratorio se observan en las tablas 5 y 6 . Los dos fucron ingresados con los diagnósticos de hepatitis viral y coma hepático. Los exámenes de laboratorio confirmaron una insuficiencia hepática aguda. El caso No 4 recibió tratamiento de coma hepático con el método conservador, corticoesteroides (Hidrocortisona $100 \mathrm{mg}$. cada 6 horas por vía endovenosa) y transfusiones por la anemia. Posteriormente por continuar febril se solicitó hemocultivo que 
T A B L A NO 3

HEIATITIS POR SALMONELLA

Reacciones de Aglutinacion y Hemocultio

\begin{tabular}{|c|c|c|c|c|c|}
\hline & Eborth $H$. & Ebarsho. & Putrat $A$ & Furat 8 & Hemoctilive \\
\hline \multirow[t]{2}{*}{1} & $1 \times 400$ & $1 \times 200$ & $1 \times 100$ & $1 \times 100$ & $(-)$ \\
\hline & $1: 400$ & $1 \times 2 \pi 0$ & $(-)$ & $1 \times 200$ & $(-)$ \\
\hline \multirow[t]{2}{*}{2} & $1 \times B C 0$ & $(-)$ & $1-j$ & $1 \times 100$ & \\
\hline & $(-)$ & $(\rightarrow)$ & $(\rightarrow)$ & $1 \times 2010$ & \\
\hline \multirow[t]{2}{*}{3} & $1 \times 50$ & $1 \times 100$ & $(-)$ & $1 \times 100$ & \\
\hline & $(-)$ & $1 \times 100$ & $(-)$ & $1 \times 200$ & {$[-]$} \\
\hline \multirow[t]{2}{*}{4} & $2 \times(0)$ & $7 \times 50$ & $(-)$ & $1 \times 100$ & \\
\hline & $1 \times 400$ & $1 \times 200$ & $1 \times 200$ & $1 \times 200$ & \\
\hline$j$ & $1 \times 160$ & $1 \times 810$ & $(-)$ & $1 \times 400$ & $(-)$ \\
\hline \multirow[t]{2}{*}{6} & $(-)$ & $(-)$ & $(-)$ & $(-)$ & Su'monella \\
\hline & $(-)$ & $(-)$ & $(-)$ & $(-)$ & Schort Müllari \\
\hline 7 & $1 \times 800$ & $1 \times 100$ & $(-)$ & $(-)$ & $\begin{array}{l}\text { Salmonella } \\
\text { Typhi }\end{array}$ \\
\hline \multirow[t]{2}{*}{8} & $\{-\}$ & $(-)$ & $(-)$ & $\{-\}$ & Salmotiella \\
\hline & $(-)$ & $(-)$ & $(-)$ & $(-)$ & BareilJy \\
\hline \multirow[t]{2}{*}{9} & $(-)$ & $(-)$ & $(-)$ & $\{\times 100$ & $(-)$ \\
\hline & $i \rightarrow$ & $(-)$ & $(-)$ & $1 \times 200$ & $(-)$ \\
\hline 10 & $1 \times 640$ & $1 \times 320$ & $(-)$ & $(-)$ & $(-)$ \\
\hline 11 & $1 \times 160$ & $1 \times 80$ & $(-)$ & $(-)$ & \\
\hline 12 & $(-)$ & $(-)$ & $(-)$ & $(-)$ & $\begin{array}{l}\text { Salmuntila } \\
\text { Bareitly }\end{array}$ \\
\hline 13 & $1 \times 40)$ & $1 \times 200$ & $(\rightarrow)$ & $1 \times 200$ & $\begin{array}{l}\text { Salmonella } \\
\text { Typhi }\end{array}$ \\
\hline \multirow[t]{2}{*}{14} & $(-)$ & $(-)$ & $(-)$ & $i-)$ & Salrronella \\
\hline & $1 \times 100$ & $1 \times 50$ & $(-)$ & $(-)$ & Typhi \\
\hline
\end{tabular}

resultó negativo y reacciones de Widal que fueron intensamente positivas. Se trató con Cloranfenicol ( $50 \mathrm{mgs} . \mathrm{Kg}$. peso día) observándose una rápida mejoría.

El caso No 12 fue hospitalizado después de 15 días de cvolución con fjebre e ictericia y en los últimos días melena. Al ingreso se constató fetor hepático y marcado compromiso de conciencia. Se diagnosticó coma hepático y se inició tratamiento con método conservador, corticoesteroides recibiendo además dos exsanguino transfusiones.

La reacción de Widal fue negativa, pero el hemocultivo dio desarrollo de Salmonella bareilly. Inicialmente recibió cloranfenicol en dosis de $\mathbf{5 0}$ mg. Kg. peso día, como no se observara mejoría posteriormente se cambió por Gentamicina $(5 \mathrm{mg}$. Kk. peso día) con lo cual la temperatura se normalizó y se evidenció una franca recuperación del estado general.

Las Salmonellosis constituyen un grupo de enfermedades agudas provocadas por agentes dcl numeroso género denominado Salmonella y que pertenecen a la familia enterobacteracea (ver tabla 7). Gran número de especies de Salmonellas son naturalmente patógenas para el hombre, ma-
TA B L A NO 4

HEPATITIS POR SALMONELLA

UK VALORES DE TGP Y BILIRRUBINA EN SUERO
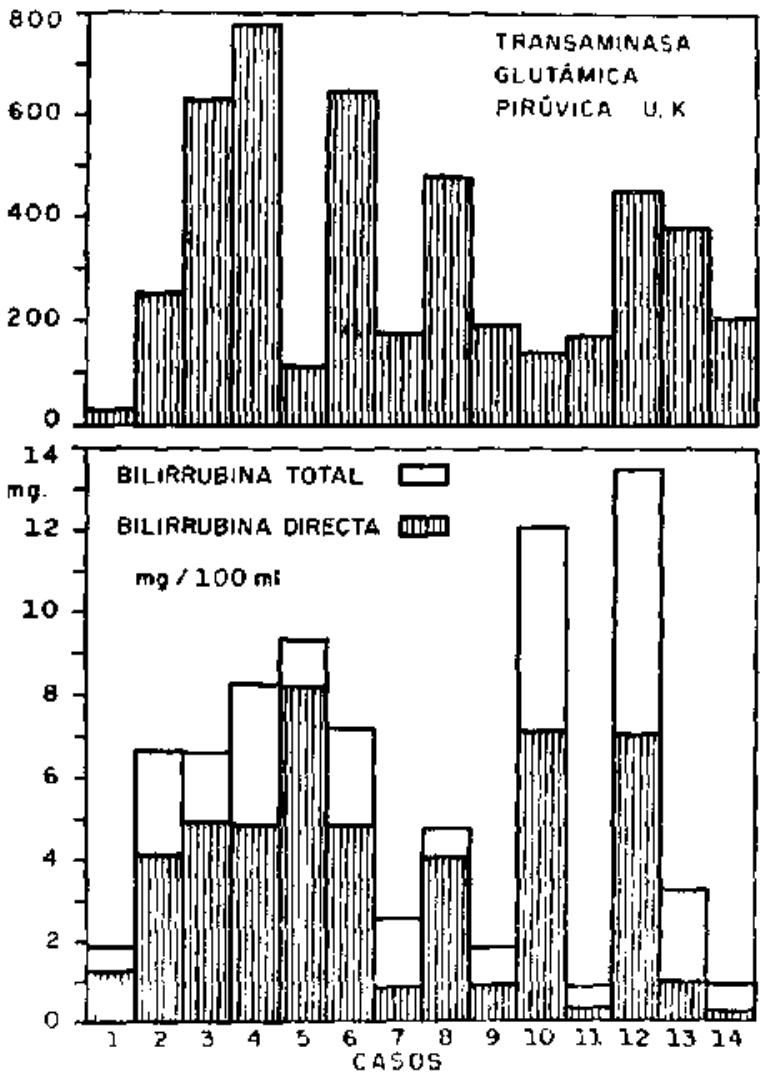

TA B LA No 5

IIEPATTIS POR SALMONELLA

Simomis en dos casos de insuficiencia heputica sute

\begin{tabular}{|c|c|c|}
\hline Caso & 4 & 12 \\
\hline $\begin{array}{l}\text { Días con fiebre } \\
\text { antes dal ingreso }\end{array}$ & 7 & 15 \\
\hline $\begin{array}{l}\text { Compromiso } \\
\text { de conciencia }\end{array}$ & + & $+t+$ \\
\hline Fctor h:pútico & $++t$ & ++ \\
\hline Icterici,a & ++ & $+-\dot{r}$ \\
\hline Hepatomegalia & $2,5 \mathrm{~cm}$ & $4 \mathrm{~cm}$ \\
\hline Hemorngia & $\stackrel{+}{\stackrel{+}{\text { (Epistusis) }}}$ & $\begin{array}{c}+++ \\
\text { (Digrstive) }\end{array}$ \\
\hline
\end{tabular}

míferos y aves. La Salmonelia typhi es patógena para el hombre y no para les demás animales. $D=1$ grupo de las Salmonellas causantes de zooncsis 
TA B L A No O

HEPATITIS POR SALMONELLA

Exämentes de Laboratorio en dos casos de insuficiencia hepatica grave

\begin{tabular}{|c|c|c|}
\hline Caso & 4 & 12 \\
\hline \multicolumn{3}{|l|}{ Bilirr ubinemia } \\
\hline Dir. Total (mg/100 ml) & $4,8 / 8,2$ & $5,7 / 13,5$ \\
\hline T. G. P. (U. K.) & 780 & 450 \\
\hline \multicolumn{3}{|l|}{ Colesterol } \\
\hline Total (mg/100 ml.) & 80 & 85 \\
\hline Protrombinemia & & \\
\hline$\%$ & 41 & 28 \\
\hline Glutamina en & & \\
\hline L.C.R. $(\mathrm{mg} / 100 \mathrm{ml})$. & 32,5 & 35,5 \\
\hline $\begin{array}{l}\text { Serinas/Globulinas } \\
(\mathrm{mg} / 100 \mathrm{ml} .)\end{array}$ & $29,6 / 42,4$ & $25,7 / 16,9$ \\
\hline Hemocultivo & $(-)$ & $\begin{array}{l}\text { Salmonella } \\
\text { Bareilly }\end{array}$ \\
\hline
\end{tabular}

Se ha observado un aumento constante del número de especies capaces de causar enfermedad en el hombre. Las infecciones humanas por Salmonella pueden adquirirse por contacto directo con animales infectados; pero es más frecuente por la ingestión de agua o alimentos contaminados. $\mathrm{Co}$ mo estos microorganismos se pueden multiplicar en la carne, leche y huevos antes de que sean consumidos, estos alimentos son los que con mayor frecuencia intervienen en la propagación de la jnfección.

En las infecciones por Salmonella se describen diversas formas clínicas (ver tabla 8), en Jos grupos 1 y 2 el bacilo penetra a través del tejido linfático intestinal y luego pasa a los ganglios mesentéricos; multiplicándose en éste, pasarian al conducto torácico y luego a la circulación general. Se localizan en los ganglios linfáticos, bazo, pulmones, médula ósea e hígado (de donde resulta la forma 4). Encontrando un buen medio de cultivo en la bilis se multiplica rápidamente en las vías biliares. En la necropsia de los casos de fiebre tifoidea se observa regularmente el hígado aumentado de tamaño y al estudio histológico se describen lesiones que varían desde una simple tumefacción turbia hasta procesos de intensa dege-

TABLA No 8

\begin{tabular}{|c|c|c|c|c|c|}
\hline \multirow[t]{2}{*}{ Grupo } & \multirow[t]{2}{*}{ Tipo } & \multirow{2}{*}{$\begin{array}{l}\text { Principales } \\
\text { antigenos o }\end{array}$} & \multicolumn{2}{|c|}{$\left.\right|_{\text {Antigenos } H .}$} & \multirow[t]{2}{*}{ Fuentes naturales } \\
\hline & & & Fase I & Fase II & \\
\hline$A$ & $\begin{array}{l}\text { 5. paratyphosa (bacilo } \\
\text { paratífico A) }\end{array}$ & II, XII & $\mathrm{a}$ & $\cdots$ & Hombre \\
\hline \multirow[t]{2}{*}{$\mathbf{B}$} & $\begin{array}{l}\text { S. schotumuetieri (bacila } \\
\text { paratífico B) }\end{array}$ & IV, XI & $\mathbf{b}$ & 1,2 & Hombre \\
\hline & $\begin{array}{l}\text { S. typhimurium (Bact. } \\
\text { aertrycke) }\end{array}$ & IV, XII & $\mathbf{i}$ & $1,2,3$ & Roedores \\
\hline \multirow[t]{4}{*}{$c_{k}$} & $\begin{array}{l}\text { S. hirschfeldit (bacilo } \\
\text { paratífico C) }\end{array}$ & $\mathrm{VI}_{3} \mathrm{VI}_{2}, \mathrm{VII}$ & $c$ & 1,5 & Hombre \\
\hline & $\begin{array}{l}\text { S. choleraesuis (S.sui- } \\
\text { pestifer tipo ameri- } \\
\text { cano) }\end{array}$ & $\mathrm{VI}_{1} \mathrm{VII}$ & c & 1,5 & $\begin{array}{l}\text { Ganado de cerdo, bovino y } \\
\text { ovino }\end{array}$ \\
\hline & S. oranienburg & $\mathrm{VI}_{1}, \mathrm{VI}_{2}, \mathrm{VII}$ & $\mathrm{m}, \mathrm{t}$ & $\cdots$ & Codornices, pollos \\
\hline & 5. monfevideo & $\mathrm{VI}_{\mathrm{i}}, \mathrm{VII}$ & $\mathrm{g}, \mathrm{ml}, \mathrm{s}$ & $\cdots$ & Monos, cerdos, pavos, polios \\
\hline $\mathrm{C}_{2}$ & S. newport & VI, VIII & $e, b$ & $1,2,3$ & Roedores, cerdos, pollos, pavos \\
\hline \multirow[t]{3}{*}{ D } & S. typhosa (bacilo túfico) & IX, XII & d & $\ldots$ & Hombre \\
\hline & $\begin{array}{l}\text { S. enteritidis (bacilo de } \\
\text { Gaertner) }\end{array}$ & IX, XII & $\mathrm{g}, \mathbf{m}$ & $\ldots$ & $\begin{array}{l}\text { Caballos, cerdos, roedores, } \\
\text { patos }\end{array}$ \\
\hline & S. pullorum & IX, XII & \multicolumn{2}{|c|}{ inmóvil } & Pollos \\
\hline E & S. anatum & III, X, XXVI & $\mathrm{e}, \mathrm{h}$ & 1,6 & Cerdos, patos, pollos, pavos \\
\hline
\end{tabular}


TA B L A No 9

CUADROS CIINICOS PRODUCIDOS POR LAS SALMONELLAS EN EL HOMBRE

1.-Ficbres entéricas: Tifoidea y paratifoidea.

2.-Septicemias.

3.-Diarrea simple - sindrome desintérico, intoxicación alimentaria.

4.-- Irfecciones localizadas: osteomielitis, meningitis, abcesos, neumonías, endocarditis.

neración granulo grasosa. Las alteraciones degerarativas paeden ser difusas o bien presentarse en forma de focos cuya extensión y densidad varía. Existen necrosis focales que se localizan en diversas zonas del lobulillo hepático e infiltrados inflamatorios de les espacios portas y nódulos tíficos que corresponden a células sinusojdales que se multiplican atrededor de células hepáticas produciendo necrosis focal.

Si bien es cierto que el compromiso hepático mínimo o moderado es frecuente en las infecciones por Salmonella, este no se manifiesta clínicamente ni tampoco a través de las pruebas funcionales hepáticas. Aquellos que dan manifestaciones clínicas de hepatitis son portadores de alteraciones histclógicas importantes (necrosis focal, núdulos tíficos, etc.).

En estos últimos años hemos comprobado una mayor frecuencia de hepatitis por Salmonella y septicenias por Salmonellas que son propias de zoonosis y yue en ciertas condiciones pueden dar origen a infecciones en el hombre, nos referimos a la Salmonella typhinurium, bareilly, newington y otras. Una de las razones que explica la aparición de estas Salmonellas en el hombre es el consumo de alimentos cárneos contaminados. Se sabe que alrededor de un $25 \%$ de los cerdos faenados en los mataderos controlados tienen Salmonellas en los ganglios (10).

Hemos visto que tanto la Salmonella typhi como otras Salmonellas pueden ocasionar un cuadro infeccioso con daño hepático importante, que puede confundirse fácilmente con una hepatitis viral. Inclus' en ocasiones puede llegar a insuficiencia hepática grave. Es aquí donde una anamnesis cuidadosa y una semiología adecuada cobran todo su valor en la orientación diagnóstica; los exámenes de laboratorio posteriormente confirman la etiologia de estas hepatitis agudas.

Debemos aceptar sí que en esta investigación no se confirmó el diagnóstico de Hepatitis tífica a través del estudio histológico hepático. Creemos que a pesar de que el cuadro clínico y la respues- ta terapśutica son compatibles con esta afección, no estamos en condiciones de rechazar la participación concomitante del virus IH en la hepatopatía de estos pacientes.

\section{RESUMEN}

Se presentan 14 pacientes con Hepatitis por Salmonella. El diagnóstico de Hepatitis se fundamentí en el cuadro clínico y exámenes de laboratorio. Los sintomas más frecuentes fueron ictericia, fiebre y hepatomegalia. Las transaminasar se encontraron elevadas en 13 de los 14 casos. Sólo en dos puctentes las reacciones de aglutinación fueron negativas, pero en ellas se aistó la Salmonella en los hemocultivos.

Dos casos evolucionaron con signos de insuficiencia hepática aguda (coma hepático), uno de ellos fue sometido a exsanguino-transfusión previo a la contirmación etiológica de su hepatopatia.

Se revisan los diversos tipos de Salmonellas en cl hombre y se llama la atención sobre la mavor incidencia de ellos en los últimos años. Aquellos que se acompañan de compromiso hepático importante pueten dar serios problemas en el diagnóstico diferencial con ta Hepatitis de etiología viral.

\section{SUMMARY}

Fourteen patients with Salnonella Iufection and acute hepatitis were studied.

The diagnosis was bascd on Clinical and laboratory findings. Jaudice, fever and hepatomegaly were th: most frecuent symptoms observed in those patients. Serum Glutamic Pirtivic Transaminase was above normal levels in thirteen casss. The agglutination reictions for Salmo. nella was recovered in blood cultures.

T'wo patients developed an acute hepatic failure. One of them wis treated with high doses of corticoest:ro:d; and exchange transfusion before the diagnosis of Salmonelliz h:patitis was established.

We call the attentiots to the bigh incidence of S.Imonella infection throughout the last 3 years in our hos. pital community and to the fact that these patients could be casily misdiagnosed as viral hepatitis when important liver demiag is present.

\section{Biblogratía}

1.-Negro R. C. y col.: Enfermedades del Hígado en la Infancia, pág. 339, Editorial Médica Pananericana B. Aires.

2.-J. Bauzá y G. Frascoli "Complicaciones Hepatovesiculares de Ja Fiebre Tifoidea en la Infancia". Rev. Chilena de Pediatría 22: 113, 1951. 
J. Bauzá y G. Frascoli: "Complicaciones Hepativesiculares de la Fiebre Tífoidea en la Infancia" $2^{\text {a }}$ parte. Rev. Chilena de Pediatría 22: 154, 1951.

3.- Sepulveda y Fremes: Complicaciones Hepatovesiculares de la Fiebre Tifoidea en la Infancia. Rev. Chilena de Pediatría 17: 667, 1946.

4.-R. Orozco y col.: Estudio Clínico de la Fiebre Tifoidea. Rev Médica de Chile 87: 266-271, 1959.

5.- M. Salcedo y col. Complicaciones y letalidad de la Febre Tifoidea y de los Paratifus A y B. Rev. Médica de Chile 95: 744, 1967.
6.-Link A. y col Hepatitis Tífica. Rev. Chilena de Pediatría 41: 832, 1970.

7.-Cachin M. "Hepatites Typho-paratyphiques" Encyclopédie Médico Chirurgicale. Foie 7015, B 30 pág. 5 .

8.-Gutiérrez, G. y Kumate J.: Un caso de Hepatitis 'Tifoídica "Bol. Méd. Hosp. Infant." (Méjico) 19: $59-63,1962$.

9.-D. Faierman et al: Complications of Thyphoid Fcver. J.A.M.A. $22 \mathrm{~L}$ : $60-61,1972$.

10.- Estudio de Salmonellas en ganglios de cerdos en mataderos de la provincia de Santiago. Tesis de Grado. Dr. Darío Pinto 1965. Universidad de Chile. 